\title{
Characteristics of particleboard manufactured from bamboo shoot sheaths
}

\author{
Surat Srichan $^{1 *}$, and Werasak Raongjant ${ }^{2}$ \\ ${ }^{1}$ King Mongkut's University of Technology North Bangkok (Prachinburi Campus), \\ Department of Construction Design and Management, Faculty of Industrial Technology \\ and Management, 129 Moo 21, Noen Hom, Muang Prachinburi, Prachinburi, Thailand \\ ${ }^{2}$ Rajamangala University of Technology Thanyaburi, Department of Civil Engineering, \\ Faculty of Engineering, 39 Moo 1, Klong 6, Khlong Luang, Pathum Thani, Thailand
}

\begin{abstract}
This study emphasizes on the physical, mechanical, and thermal properties of single-layer particleboard manufactured from bamboo shoot sheaths. Particleboards were produced through the hot-pressed process and glued together by Diphenylmethane Diisocyanate (MDI) adhesive. This is in order for producing specified densities of boards as $400-\mathrm{kg}, 600-\mathrm{kg}$, and $800-\mathrm{kg}$ per $\mathrm{m}^{3}$. The raw material was sieved into four sizes: No.1-No.4 mesh. The particleboards were used to find board density, water absorption, thickness swelling, modulus of elasticity, modulus of rupture in bending, and internal bonding according to the JIS standard. Coefficients of thermal conductivity (k) of boards were discovered by a heat flow meter in steadystate conditions. The results showed that the particleboard performed outstandingly in terms of tensile strength perpendicular to the surface (internal bonding) and low thermal conductivity. However, there were some weaknesses found from their characteristics that were high water absorption and thickness of swelling, low modulus of elasticity and modulus of rupture comparing to standard criteria. The results also indicated that bamboo shoot sheaths, the agricultural residue, can be produced as particleboard, and are appropriate for an indoor heat insulator, but further investigation is required to improve the strength and durability of the particleboard.
\end{abstract}

\section{Introduction}

Thailand is an agricultural country and most of the agricultural product sources and exports. The agricultural waste in Thailand is widespread, and it also comes from many sources. A number of agricultural wastes are based on all agricultural products of the country. There is still no utilization for some types of agricultural residues: such as green tops and dry leaves of sugarcane, cassava stumps, bamboo shoot sheaths, etc. The recycling of agricultural residues presents a viable alternative to prevent those residues from ending up with inappropriate final disposal in landfills or open-air burning, which can cause negative environmental consequences such as the greenhouse effect. An interesting choice of recycling agricultural residues is manufacturing particleboards.

*Corresponding author: surat.s@fitm.kmutnb.ac.th 
Particleboards are products composed of wood particles bonded with synthetic adhesives that are consolidated under heat and pressure. Currently, an alternative for particleboard production may be the use of agricultural lignocellulosic waste to replace solid wood particles in the manufacturing of panels. Several researchers have studied particleboard manufacturing from agricultural residues. [1] studies properties of panel boards from coconut fibers and PET bottles. The overall results show that a coconut fiber panel board is better than a conventional panel board since the coconut fiber panel board is less swelling, it also has lower water absorption, high modulus of rupture and low modulus of elasticity. Furthermore, [2] evaluates particleboard from sugarcane bagasse and bamboo leaves particles to be used as construction products and flooring in Brazil. The result indicates that a sugarcane bagasse particleboard which is medium-density $\left(870 \mathrm{~kg} / \mathrm{m}^{3}\right)$ with $12 \%$ content of castor oil bicomponent adhesive has superior performance than a bamboo leaves-based board. [3] assesses the physical-mechanical properties of particleboards manufactured with wood (Eucalyptus grandis), bamboo (Bambusa vulgaris), and/or rice husk particles, these were separated or combined in different proportions. The findings reveal that most parameters of the particleboards manufactured exclusively with wood particles show similar performance to bamboo and wood-bamboo particleboards.

Bamboo occurs mostly in tropical and sub-tropical zones. It has two distinctive parts: a stem and a rhizome. The stem, called the culm, is the upper ground part of bamboo that contains most of the woody material. The bamboo shoot is the part that grows from the underground rhizome. A shoot's sheath is what envelops the bamboo pulp. The outer surface of the sheath is rough and hairy, and the inside is a glossy texture covering it. Bamboo is one of the non-wood materials that can be utilized as raw material for making particleboard since it is a lignocellulosic material with a high amount of lignin, cellulose, and fiber [4]. Moreover, there are several advantages of bamboo compared to other woody plants, one of them is that bamboo plants grow rapidly, easy to plant and reproduce, highly productive and easy to harvest. Bamboo chip consists of $22.4 \%$ lignin, $19.5 \%$ xylans, $49.3 \%$ cellulose, $16.8 \%$ extractive and 1.5\%-3\% ash [5]. Therefore, many researchers have developed the use of bamboo culm as strips, chips, planer waste, powder, and wool of particle shape with boards manufactured for various purposes, such as the use of composite boards, particleboards, laminated sheets, veneer boards, and acoustic boards [6-12]. They have mostly studied the physical and mechanical characteristics of their boards. These results indicate that the main mechanical properties of bamboo boards produced from the culms meet international standards for wooden boards. However, water absorption and thickness swelling still need to be improved.

Besides, the effect of product type and density on the thermal properties of engineered bamboo-composite panels was specifically investigated. [6] finds that material density has a clear and more substantial influence on thermal conductivity because increasing panel density also increases thermal conductivity. Likewise, researchers investigate the effect of the insulation material's different densities on the additional heat transferred within a typical wall of a residential building [13]. The results report that the optimum density level is UHD $\left(30 \mathrm{~kg} / \mathrm{m}^{3}\right)$ among the four levels of the EPS density as the UHD polystyrene releases the lowest amount of heat to the building interior.

As previously stated, no work has been done that a researcher studies the properties of particleboard made of bamboo shoot sheaths. Consequently, the author emphasizes an interest in using the residue sheaths of bamboo shoots to produce particleboard; then, it is tested for physical, mechanical, and thermal properties to consider if it suits usage and is efficient enough to replace wood particleboard. 


\section{Materials and methods}

\subsection{Raw material collection and preparation}

Shoot sheaths of the Dendrocalamus Asper bamboo, the residues by the canned shoot manufacturing in Thailand's locality, were gathered for this research.

Preparing raw materials for produced particleboards had the following steps. Firstly, the shoot sheaths were desiccated in the sun to reduce humidity to be appropriate for the process. Then, these shoot sheaths were ground into the least particle size about several times via two different types of grinders. The first one is the wood chipper machine that uses blades to reduce the size of pieces; as a result, the particles become scraps entangled with long fibers. The latter is the hammer mill machine used to reduce particle size by pulverizing; thus, the sheath scraps become smaller and shorter. The process was repeated until the particle size was smaller than $2 \mathrm{~mm}$. The particles obtained were classified using a vibrating machine and sieves of No.1 mesh $(1.0 \mathrm{~mm})$, No.2 $(0.6 \mathrm{~mm})$, No.3 $(0.5 \mathrm{~mm})$, and No.4 (smaller than 0.5 $\mathrm{mm}$ ). This study used sheath particles of $0.5-\mathrm{mm}$ and $0.6-\mathrm{mm}$ size to produce particleboards for physical and mechanical properties test, as shown in Figure 1. The particleboards made of all four particle sizes used to test for thermal conductivity.

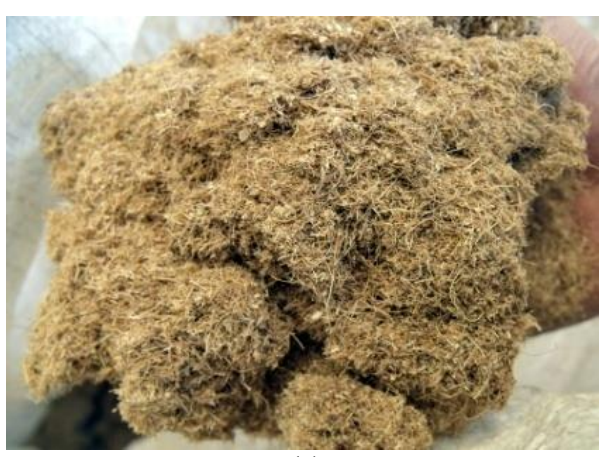

(a)

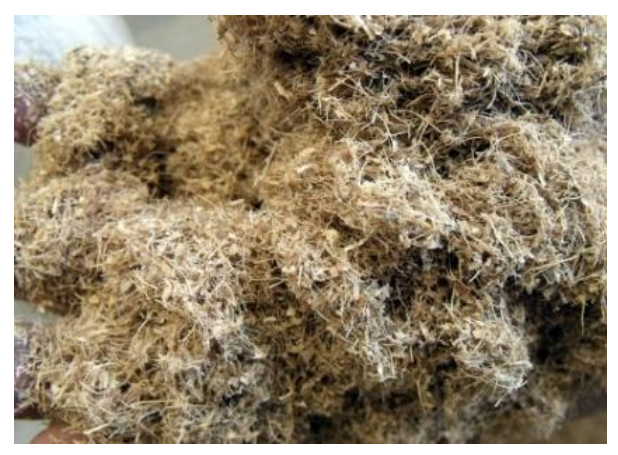

(b)

Fig. 1. The feature of bamboo shoot sheath particles with (a) $0.5-\mathrm{mm}$ and (b) $0.6-\mathrm{mm}$ size.

\subsection{Particleboard manufacturing}

Single-layer particleboards with a size of $400 \times 400 \times 10 \mathrm{~mm}^{3}$ and target medium densities of $400 \mathrm{~kg} / \mathrm{m}^{3}, 600 \mathrm{~kg} / \mathrm{m}^{3}$, and $800 \mathrm{~kg} / \mathrm{m}^{3}$ were produced, respectively. Diphenylmethane Diisocyanate (MDI) resin was used as the binder for particleboard manufacturing with 7\% resin content based on the weight of the dried particle. The particles were then blended in a rotary drum mixer with MDI resin which was slowly sprayed into the mixer while it rotated. The glued particles were then spread manually into a mat-forming box of $400 \mathrm{~mm} \times 400 \mathrm{~mm}$ $\times 10 \mathrm{~mm}$. The mat was then pressed at the pressure of $25-30 \mathrm{~kg} / \mathrm{cm}^{2}$ and $120-125^{\circ} \mathrm{C}$ for 5 $\mathrm{min}$. All boards were conditioned at room temperature $\left(31^{\circ} \mathrm{C}\right)$ and $65 \%$ relative humidity for one week before testing. Eventually, the samples were tested according to the JIS standard applied to the boards: such as density, moisture content, water absorption, thickness swelling, modulus of elasticity, modulus of rupture, internal bonding, and thermal conductivity. 


\subsection{Properties testing}

For preparing panels for testing, the samples are cut into pieces with an area of $50 \mathrm{~mm} \times 50$ $\mathrm{mm}$ for water absorption, thickness swelling, and internal bonding test while cutting the samples into pieces with an area of $200 \mathrm{~mm} \times 50 \mathrm{~mm}$ for modulus of rupture and modulus of elasticity test. Moreover, the samples cut with an area of $300 \mathrm{~mm}$ x $300 \mathrm{~mm}$ are used for the thermal conductivity test. The tests to find the physical and mechanical properties of the particleboards were achieved under the JIS A5908-2003 standard. The thermal conductivity test of the boards was tested by Heat Flow Meter performed in steady-state conditions with the HFM 436/3 Lambda device. Overall, 30 particleboards were produced: 12 ones for the thermal test, the other ones for the physical and mechanical test.

\section{Results and discussion}

The pulverized shoot sheaths were analysed the particle size with a microscope, by sieving them through a mesh strainer to get 100 pieces of sheaths; then, their width, length, and thickness were measured. The results revealed that at $74 \%$ of all the particles, No.2-mesh $(0.6 \mathrm{~mm})$ and No.3-mesh $(0.5 \mathrm{~mm})$ of particle size had the slenderness ratio (length/thickness) around 12.1-15.7 and 9.3-15.2, respectively. The results pointed out that the No.2-particle is longer and thinner than the No.3-particle. However, both sizes were considered as short and thick. The test results of physical, mechanical, and thermal properties could be concluded as follows.

\subsection{Physical properties of particleboard}

\subsubsection{Density and moisture content}

The medium density particleboards had higher density than the criteria given in the experiment, with values around $554-836 \mathrm{~kg} / \mathrm{m}^{3}$ and $475-889 \mathrm{~kg} / \mathrm{m}^{3}$ of No.2-mesh $(0.6 \mathrm{~mm})$ and No.3-mesh $(0.5 \mathrm{~mm})$ particles, respectively. The density value depended on the quantity of sheath particles in the mixture. The moisture content (MC) increased along with quantities of added particles. The density of particleboard and the size of sheath particles affect insignificantly the moisture content [10]. The approximate $7.8 \%-8.9 \%$ of water content complies with the JIS's criteria standard regulating moisture content in the range of 5\%-13\% [14]. The density and moisture content of particleboards in each particle size are as shown in Figure 2.

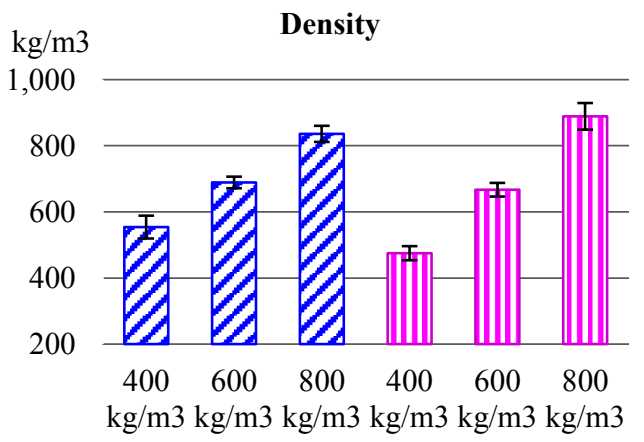

No. 2

No. 3

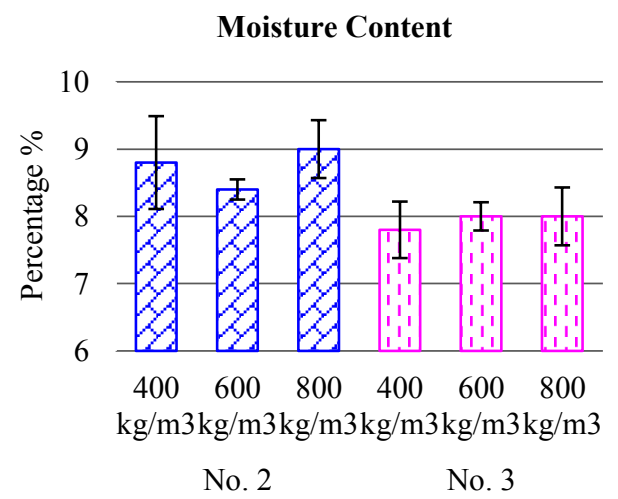

Fig. 2. Means with $95 \%$ confidence intervals of density and moisture content of the particleboards. 


\subsubsection{Water absorption and thickness swelling}

Figure 3 shows the water absorption $(24 \mathrm{~h})$ and thickness swelling $(24 \mathrm{~h})$ values of the particleboards at different densities and particle sizes. The water absorption (WA) increases when the board density decreases since there are lots of pores to contain water into the gaps throughout the board. The JIS standard has no referential value for water absorption [14]. However, similar results are observed by [9] and [2] at the $600 \mathrm{~kg} / \mathrm{m}^{3}$ and the $800 \mathrm{~kg} / \mathrm{m}^{3}$ of particleboard density, respectively. When the particle size was considered with the same board density, it was found that the no.3-mesh $(0.5 \mathrm{~mm})$ of a particle could absorb more water than the no.2-mesh $(0.6 \mathrm{~mm})$. Since many small particles have higher surface area and more empty spaces, allow themselves to absorb much water [10].
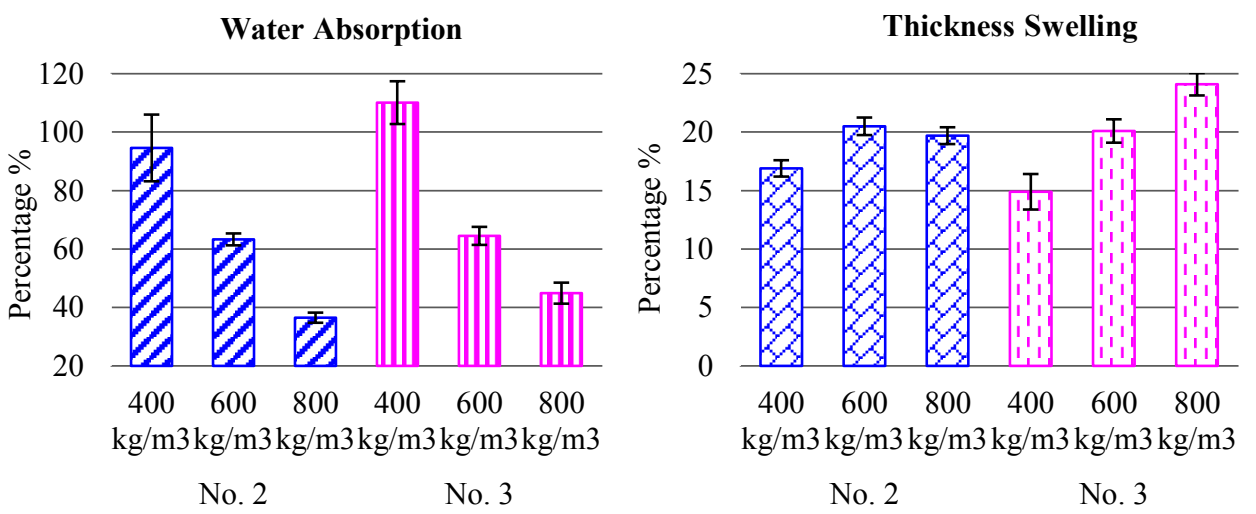

Fig. 3. Means with $95 \%$ confidence intervals of water absorption and thickness swelling of the particleboards.

The thickness value of swelling (TS) submerging in water for 24 hours depended on the boards' density. When the particleboard density increased, the TS value was also increased due to the increased thickness of many particles within the particleboard. These particles are mostly composed of celluloses and lignin which causes much hydrophilic behavior [3]. This results in the severe swelling of the immersed particleboard. When compared to the JIS standard criteria [14], it was found that all densities do not pass the swelling criterion (12\% of maximum). It was explained due to using only an adhesive without paraffin during the particleboard-manufacturing procedure, according to results from [15]. However, the results showed that the TS value was indistinctly affected by particle size.

\subsection{Mechanical properties of particleboard}

\subsubsection{Modulus of elasticity and modulus of rupture to static bending}

Figure 4 shows the result of the bending test, it points out that modulus of elasticity (MOE) and modulus of rupture (MOR) depend on the particleboard's density. When the board is denser, the MOE and the MOR value also increase. Considering the particle size, it can see that No.3-sized particle being smaller than No.2-sized particle mostly gives better MOE and MOR values. On the other hand, [10] showed MOE and MOR values of particleboards made from chips of Dendrocalamus Asper bamboo culms are higher than the values of sheathbased particleboards at similar density. It possibly states that the shoot sheath particleboard provides less flexural strength than those made out of the bamboo culms. Also, when the 
MOE and MOR values were compared to the JIS standard stipulated a minimum of 3,000 $\mathrm{MPa}$ and $18 \mathrm{MPa}$, respectively[14]. It was found that most of the densities of the particleboard had lower MOE and MOR values than the criteria except for the board with $800 \mathrm{~kg} / \mathrm{m}^{3}$ of density providing MOR value according to the standard criteria. Therefore, it indicates that the utilization of particleboard made from the bamboo shoot sheath can be suitable for the non-structural work, which relates to [9]'s comment.

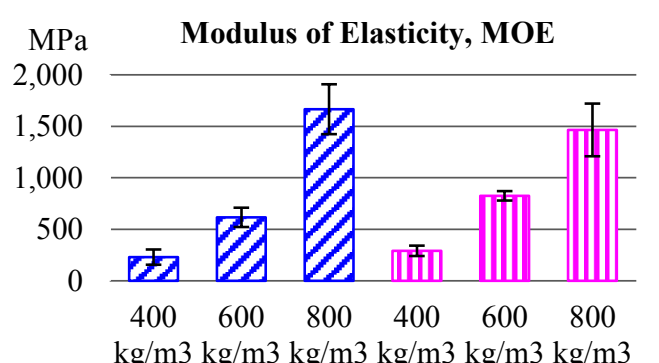

No. 2

No. 3

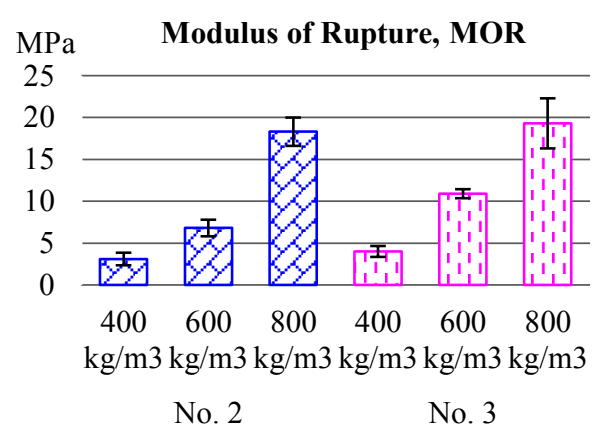

No. 2

Fig. 4. Means with $95 \%$ confidence intervals of modulus of elasticity and modulus of rupture of the particleboards.

\subsubsection{Internal bonding}

Figure 5 shows the test result of tensile strength perpendicular to the surface (Internal Bonding) whose value varies according to the board's density. The internal bonding (IB) value increases while the density of board increases. When considering the particle size's effect on the particleboard based on shoot sheaths, it could be found that it did not significantly affect the IB value, relating to research results of [10]. Other than that, when compared to JIS standard's criteria [14], it was found that all the density of particleboard met the JIS standard criterion stipulated more than $0.30 \mathrm{MPa}$ of IB value. This study showed obviously that the IB values are higher than other researches using bamboo culms as the raw material in particleboard manufacturing $[2,3,7,10,15]$. The hypothesis to the reason for the high IB value may be the result of efficiently bonding between bamboo particles and MDI glue. In addition, the internal bonding property reveals the degree of adhesive bonding among particles [10]. Therefore, it could be summarized that bamboo shoot sheath has a property that allows it to bond with MDI glue efficiently.

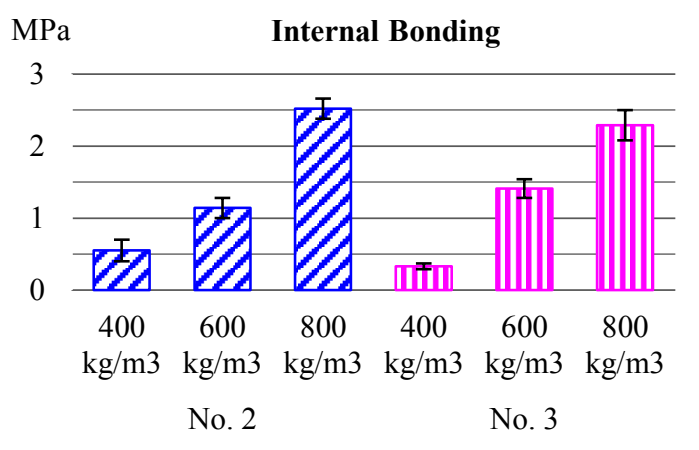

Fig. 5. Means with $95 \%$ confidence intervals of internal bonding of the particleboards. 


\subsection{Thermal properties of particleboard}

To be a good heat insulator, there should be low coefficient of thermal conductivity (k) or high thermal resistance (R). The results shown in Figure 6 illustrate that the potential for boards to be a heat insulator depends on the particleboards' density. The lower density boards are more efficient at insulating heat than the higher density ones. This is due to lower density boards have a higher number of air gaps (relative insulator) inside the board than the content of particles (relative conductors), and consequently lowering the thermal conductivity [6]. Besides, particleboards from shoot sheaths produced with $400-800 \mathrm{~kg} / \mathrm{m}^{3}$ of density gave the thermal conductivity coefficient $(\mathrm{k})$ at around $0.066-0.129 \mathrm{~W} / \mathrm{m} \cdot \mathrm{K}$. The coefficient of thermal conductivity acquired from this study was then compared to the study from [6]. The researcher has studied the thermal conductivity of four different engineered bamboo products, getting the $\mathrm{k}$ value around $0.20-0.35 \mathrm{~W} / \mathrm{m} \cdot \mathrm{K}$ at $626-960 \mathrm{~kg} / \mathrm{m}^{3}$ of density. The comparison result points out that sheath-based particles can insulate heat better than engineered bamboo products made of chips, strips, or strands of bamboo.

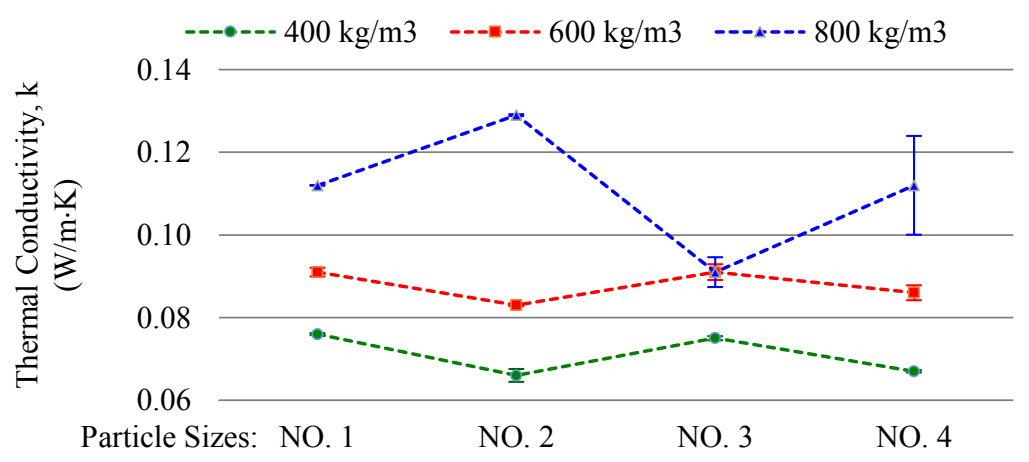

Fig. 6. Means with standard deviation bars of thermal conductivity of the particleboards.

Comparing thermal property of the particleboard with heat insulators found in the market such as fiberglass, mineral wool, polyurethane, etc., $0.017-0.045 \mathrm{~W} / \mathrm{m} \cdot \mathrm{K}$ of thermal conductivity, it was found that the particleboard has less efficiency as a heat insulator. However, it is a better overall insulator than partition wall materials, such as fiber cement boards, plywood, gypsum boards, clay bricks, concrete blocks, etc., $(0.089-0.519 \mathrm{~W} / \mathrm{m} \cdot \mathrm{K}$ of thermal conductivity). Furthermore, the increase of particleboard's heat insulation capabilities depends on the thickness of boards. With thicker particleboards, its heat insulation capabilities are also increased.

\section{Conclusions}

This study focuses on the characteristics of particleboard manufactured from bamboo shoot sheaths with the hot-pressed process using Diphenylmethane Diisocyanate (MDI) glue to bond the material and producing particleboards of $10 \mathrm{~mm}$ thickness at test densities of 400$\mathrm{kg}, 600-\mathrm{kg}$, and $800-\mathrm{kg}$ per $\mathrm{m}^{3}$. The raw material was sieved into 4 different sizes: No.1mesh, No.2, No.3, and No.4. Particles sized No.2 and No.3 were used for the board's physical and mechanical property tests. The thermal conductivity test was achieved all four sizes.

The physical and mechanical properties of particleboards made with bamboo shoot sheath residues passed JIS's standard as follows: density, moisture content (MC), and internal 
bonding (IB) values whereas boards did not pass the JIS standard on thickness swelling (TS), modulus of elasticity (MOE), and modulus of rupture (MOR) except the board at $800 \mathrm{~kg} / \mathrm{m}^{3}$ of density passed the MOR criterion. Although the JIS standard did not assign any criteria for water absorption (WA) value, the particleboards showed high water absorption values because there was no addition of paraffin wax into the mixture. Further study on particleboards' thermal conductivity revealed that the coefficient of thermal conductivity (k) is distinctly lower than boards made from culms of bamboo. The particleboard also has better heat resistance than conventional wall materials although it has poorer than commercial insulation products.

The results also indicated that the bamboo shoot sheaths, being a type of agricultural residues, can be produced as particleboard, and are also appropriate for being an indoor heat insulation material. However, further investigation of the particleboard is required to improve strength and durability. For future work, it is advised to investigate that factors affect the performance of particleboard made from bamboo shoot sheaths, particularly resin content, size and morphology of the bamboo shoot sheath.

The author would like to express their gratitude to all parties who have contributed to this successful research, especially to the Faculty of Industrial Technology and Management of KMUTNB's Prachinburi Campus and King Mongkut's University of Technology North Bangkok.

\section{References}

1. N. Ngadiman, M. Kaamin, A. Abd. Kadir, S. Sahat, A. Zaini, S.R. Nor Zentan, et al., E3S Web Conf, 34, 01014, (2018)

2. R.A.G. Battistelle, D.M. Fujino, A. Silva, B.S. Bezerra, I.D. Valarelli, J Sustain Dev Energy Water Environ Syst-JSDEWES, 4, 408, (2016)

3. R.R. de Melo, D.M. Stangerlin, R.R.C. Santana, T.D. Pedrosa, Mater Res-Ibero-am J Mater, 17, 682, (2014)

4. X. Li, Physical, Chemical, and Mechanical Properties of Bamboo and Its Utilazation Potential for Fiberboard Manufacturing, MSc. Thesis, Louisiana State University and Agriculture and Mechanical College, (2004)

5. S. Hidayati, E. Suroso, W. Satyajaya, D.A. Iryani, IOP Conf Ser Mater Sci Eng, 532, 012024, (2019)

6. D.U. Shah, M.C.D. Bock, H. Mulligan, M.H. Ramage, J Mater Sci, 51, 2991, (2016)

7. D. Biswas, S. Kanti Bose, M. Mozaffar Hossain, Int J Adhes Adhes, 31, 84, (2011)

8. Y. Ratna Pratiwi, I. Widiastuti, B. Harjanto, E3S Web Conf, 130, 01040, (2019)

9. I.D. Valarelli, R.A.G. Battistelle, M.A.P. Bueno, B.S. Bezerra, C.I. de Campos, M.C.D. Alves, Materia, 19, 11528, 1, (2014)

10. J.T.D. Bazzetto, G. Bortoletto, F.M.S. Brito, Floresta Ambient, 26, e20170125, (2019)

11. Y.F. Zhang, J. Zheng, H.L. Guo, Y.W. Li, M.G. Lu, J Appl Polym Sci, 132, 42280, (2015)

12. L. Karlinasari, D. Hermawan, A. Maddu, B. Martianto, I.K. Lucky, N. Nugroho, et al., BioResources, 7, 5700, (2012)

13. M. Khoukhi, A. Hassan, S. Abdelbaqi, E3S Web Conf, 103, 02001, (2019)

14. Japanese Standards Association, JIS A5908:2003 (English):Particleboards, Japanese Industrial Standards Committee, Japan, (2003) 
15. O. Nurhazwani, M. Jawaid, P.M. Tahir, J.H. Abdul, S.A. Hamid, BioResources, 11, 306, (2016) 